\title{
Point process methods in epidemiology: application to the analysis of human immunodeficiency virus/acquired immunodeficiency syndrome mortality in urban areas
}

\author{
Jose Antonio Quesada, ${ }^{1}$ Inmaculada Melchor, ${ }^{2}$ Andreu Nolasco ${ }^{1}$ \\ ${ }^{1}$ Research Unit on Mortality Analysis and Health Statistics, Department of Community Nursing, Preventive \\ Medicine and Public Health and History of Science, University of Alicante, Alicante; ${ }^{2}$ Registro de \\ Mortalidad de la Comunitat Valenciana, Servicio de Estudios Epidemiológicos y Estadísticas Sanitarias, \\ Subdirección General de Epidemiología y Vigilancia de la Salud, Conselleria de Sanitat, Generalitat \\ Valenciana, Alicante, Spain
}

\begin{abstract}
The analysis of spatio-temporal patterns of disease or death in urban areas has been developed mainly from the ecological stud-

Correspondence: Jose Antonio Quesada, Research Unit on Mortality
Analysis and Health Statistics, Department of Community Nursing,
Preventive Medicine and Public Health and History of Science,
University of Alicante, Carretera San Vicente del Raspeig s/n. 03690,
San Vicente del Raspeig-Alicante, Spain.
Tel: +34.965903919 - Fax: +34.965903964.
E-mail: ja.quesada@ua.es
\end{abstract}

Key words: Point process; Mortality; Urban areas; HIV/AIDS; Spain.

Ethical considerations: due to the characteristics of this observational study based on administrative retrospective data, in Spain this research needs not be approved by an ethics committee because it meets the requirements of the Spanish data protection law, and does not pose ethical problems because the researchers have only had access to anonymous data without any identification of the individuals. The anonymous databases were constructed by authorised staff from the Mortality Register of the Valencian Community for cases, and by authorised staff from the SIP for controls, according Spanish data protection law. The addresses were geocoded in geographic coordinates by the authors. Due to the fact that the databases were anonymous and the large scale of the study area, it was not possible to identify a person from the geocoding process or in the results of this study, thus protecting confidentiality. The Mortality Register and the SIP Register of the Valencian Community are not publicly available and both are subject to statistical confidentiality. However, some institutions like Alicante University are authorised to request and use anonymous databases for research purposes.

Received for publication: 8 September 2016.

Revision received: 19 April 2017.

Accepted for publication: 20 April 2017.

CCopyright J.A. Quesada et al., 2017

Licensee PAGEPress, Italy

Geospatial Health 2017; 12:506

doi:10.4081/gh.2017.506

This article is distributed under the terms of the Creative Commons Attribution Noncommercial License (CC BY-NC 4.0) which permits any noncommercial use, distribution, and reproduction in any medium, provided the original author(s) and source are credited. ies approach. These designs may have some limitations like the ecological fallacy and instability with few cases. The objective of this study was to apply the point process methodology, as a complement to that of aggregated data, to study the human immunodeficiency virus/acquired immunodeficiency syndrome (HIV/AIDS) mortality in men in the city of Alicante, Spain. A case-control study in residents in the city during the period 20042011, divided into two periods 2004-2007 and 2008-2011 was designed. Cases were men who had died from HIV/AIDS with the controls represented by the general population, matched by age to cases. The risk surfaces of death over the city were estimated using the log-risk function of intensities, and we contrasted their temporal variations over the two periods. Significant high risk areas of death by HIV/AIDS coinciding with the financially most deprived areas in the city were detected, while a statistically significant spatial change of areas at risk between the two periods studied was not found. The point process methodology is a useful tool to analyse the patterns of death by HIV/AIDS in urban areas.

\section{Introduction}

For the last few years, the number of analyses of spatial and temporal patterns in cases of disease and death in urban areas has increased. The development of statistical methods for detecting clusters and disease mapping have evolved, especially by applying them to aggregate data in ecological studies. Generalized linear mixed models have been widely used for smoothing in small city areas, particularly using a Bayesian approach, e.g., the Besag, York and Mollié model (1991) or relative risk mixed models (Besag et al., 1991; Lawson and Clark, 2002). These designs and models have certain advantages but there are also some limitations, for instance the well-known ecological fallacy, i.e. the difficulty to establish an exposure-disease association at the individual level. Another limitation might be the adjustment of potentially confounding variables, as well as the difficulty to estimate a model when there are few cases. This is the case for rare or infrequent diseases or causes of death. Richardson et al. (2004), highlights that usual Bayesian models for smoothing disease risk may have certain estimation biases: they are conservative, since they have a low sensitivity to detect risk areas with a small excess risk. As false alarms are avoided, this may be considered an advantage. Nevertheless, in these cases the risk of disease or death is underestimated and, since most of the risks due to environmental factors 
are low, these methods are hardly suited to estimate them. Additionally, Gelman and Price (1999) have shown that estimations may be affected and spurious results obtained if there is high variability in sample sizes between regions.

Mortality due to the human immunodeficiency virus/acquired immunodeficiency syndrome (HIV/AIDS) is now considered as a preventable cause of death. It is included, among other causes of death, in an indicator of potential problems to be investigated during primary prevention, both through the promotion and protection of health and through health education (Nolte and McKee, 2004). Since the introduction of antiretroviral therapy in the mid-1990s, HIV/AIDS mortality has shown a declining trend both in Europe and Spain (Borrell et al., 2006; Mackenbach et $a l ., 2013)$. In spite of this, it is of great interest to analyse preventable causes of death in urban areas, particularly HIV/AIDS, due to, on the one hand, the associated risk determinants and factors, and on the other, the fact that it is estimated that half of the European population resides in municipalities with more than 50,000 inhabitants (Raskin and Kemp-Benedict, 2004). Detecting areas with higher risk of death due to HIV/AIDS in these urban areas provides information essential for design and implementation of efficient actions and prevention plans concerning morbidity and mortality due to this syndrome. In this regard, several studies conducted in urban areas have revealed that there are still socioeconomic inequalities in HIV/AIDS mortality, although they show a decreasing trend (Borrell et al., 2006; Nolasco et al., 2009; Regidor et al., 2011; Richardson et al., 2014; Salgado-Barreira et al., 2014). Nevertheless, all these studies were conducted by means of aggregate data in areas of the urban cores studied (census tracts, quarters, districts, etc.).

Point process methods are a different approach to detect disease/death clusters in urban areas. These methods are based on the spatio-temporal analysis of the patterns of occurrence of individual events (disease/death) in the region of study and during the period of study; therefore, they are free from administrative boundaries and from the limitations and potential biases of the aforementioned methods for aggregate data. Point processes have many applications and have also been used in different contexts in different studies. In the epidemiological field, they have been used to study Legionella infection in Glasgow and Edinburgh (Diggle and Chetwynd, 1991), lung and laryngeal cancer in Lancashire, UK (Kelsall and Diggle, 1995), incidence of childhood leukaemia (Birch et al., 2000; Wheeler, 2007), asthma diagnosis (Diggle and Rowlingson, 1994), early detection of peak incidence of gastrointestinal infections (Diggle et al., 2005a), bovine tuberculosis in a county in UK (Diggle et al., 2005b), foot and mouth disease epidemic in UK in 2001 (Diggle, 2006) or the incidence of Campylobacter infections in humans in northern England (Gabriel and Diggle, 2009) among many other applications.

The application of point processes to the analysis of mortality is scarce. Nevertheless, these methods have been applied to study the sudden infant death in North Carolina (Kulldorff, 1997), the study on mortality by lung, stomach and pancreatic cancer in Walsall, UK (Kelsall and Diggle, 1998), the analysis of multiplecause deaths in the city of Tandil, Argentina (Linares et al., 2008) or the study on mortality by cancer in the city of Perth, Australia (Shao, 2011). In Spain, the mortality by cancer of the pleura and peritoneum in the city of Madrid has been analysed (LópezAbente and Ibáñez, 2001). However, no study conducted through point processes has been found on HIV/AIDS mortality.

Due to the aforementioned limitations of methods for aggregate data (especially infrequent causes of morbidity or mortality) and also due to the interest in HIV/AIDS mortality patterns in urban areas, where no studies using point process methods have been carried out, this study aimed to apply the point process methodology to the analysis of HIV/AIDS mortality in the municipal area of the city of Alicante, Spain during two time periods (2004-2007 and 2008-2011) as a complementary approach to aggregate data methods. This city, with a population of about 330,000 inhabitants, ranks among the 11 most populous cities in Spain and represents therefore a medium-sized city. The specific objectives were two-fold: to detect death risk areas in the city during each period of study by identifying areas with significantly high or low risk; and to analyse the temporal variation of the estimated risk areas between the periods of study.

\section{Materials and Methods}

\section{Study area and cases}

This was a case-control study to analyse mortality trends in the city of Alicante between 2004 and 2011. The cases involved were resident men of Alicante who had died from HIV/AIDS between 2004 and 2011. The area analysed was a closed area of approximately $176 \mathrm{~km}^{2}$ comprising the municipal area of Alicante (Figures 1 and 2).

Mortality data were obtained from the Mortality Register of the Valencian Community in Spain, which is the region where Alicante is located. The data used include all deaths coded as B20-B24 and R75 in the International Classification of Diseases, $10^{\text {th }}$ version (ICD-10), occurred to residents in the area of study. Men of all ages were included. Individuals residing in institutions (retirement homes, hospitals, prisons, etc.) or having addresses that were incomplete or did not belong to the region of study were excluded. Two different periods were distinguished: the first period covered 2004-2007 and second 2008-2011. Table 1 shows the number of cases (deaths) by age group and period.

In order to compare the spatio-temporal patterns of these cases with the ones of the population at risk, some random samples from the lists of living individuals of the Population Information System (SIP) register were taken as control groups. This register is produced by the health authority of the Valencian Community and includes the population residing in said region. The SIP register is updated every year. Controls were established by matching the cases by age (five-year age groups) and sex on a 10 to 1 ratio of controls to cases (Wheeler, 2007). Two control groups were chosen, one for each period made up by individuals still living during the last year of each period, with the same inclusion and exclusion criteria as for the cases.

The variables collected were age, sex, residential address (street and number) for cases and controls, and date of death of each case. Residential addresses were geocoded in geographic coordinates through Google geocoding API - (Application Programming Interface) v.3 (Google, 2014; Quesada et al., 2013), and were converted into Universal Transversal Mercator (UTM) coordinates. 


\section{Statistical analysis}

Let spatial point process be the stochastic process that generates space events in a region $A$, which is flat and finite. The events generated by the underlying point process of the cases and controls in the region $A$ in each period of study are $\left\{\mathrm{x}_{\mathrm{i}}\right\},\left\{\mathrm{y}_{\mathrm{i}}\right\} \in A \subset \mathfrak{R}^{2}$, $i=1, \ldots, n$, respectively. The intensity functions of the cases and controls in any location $x$ of $A$ are $\lambda_{1}(x)$ and $\lambda_{2}(x)$, respectively. The intensity function means the expected number of events per unit area and is the informal equivalent of the concept rate in epidemiology when time is replaced by space. Intensities are supposed to be inhomogeneous in $A$, i.e. they vary depending on the location $x$ where the evaluation is conducted due to the space variation of the population at risk in $A$. Thus, these two processes are considered as inhomogeneous Poisson point processes.

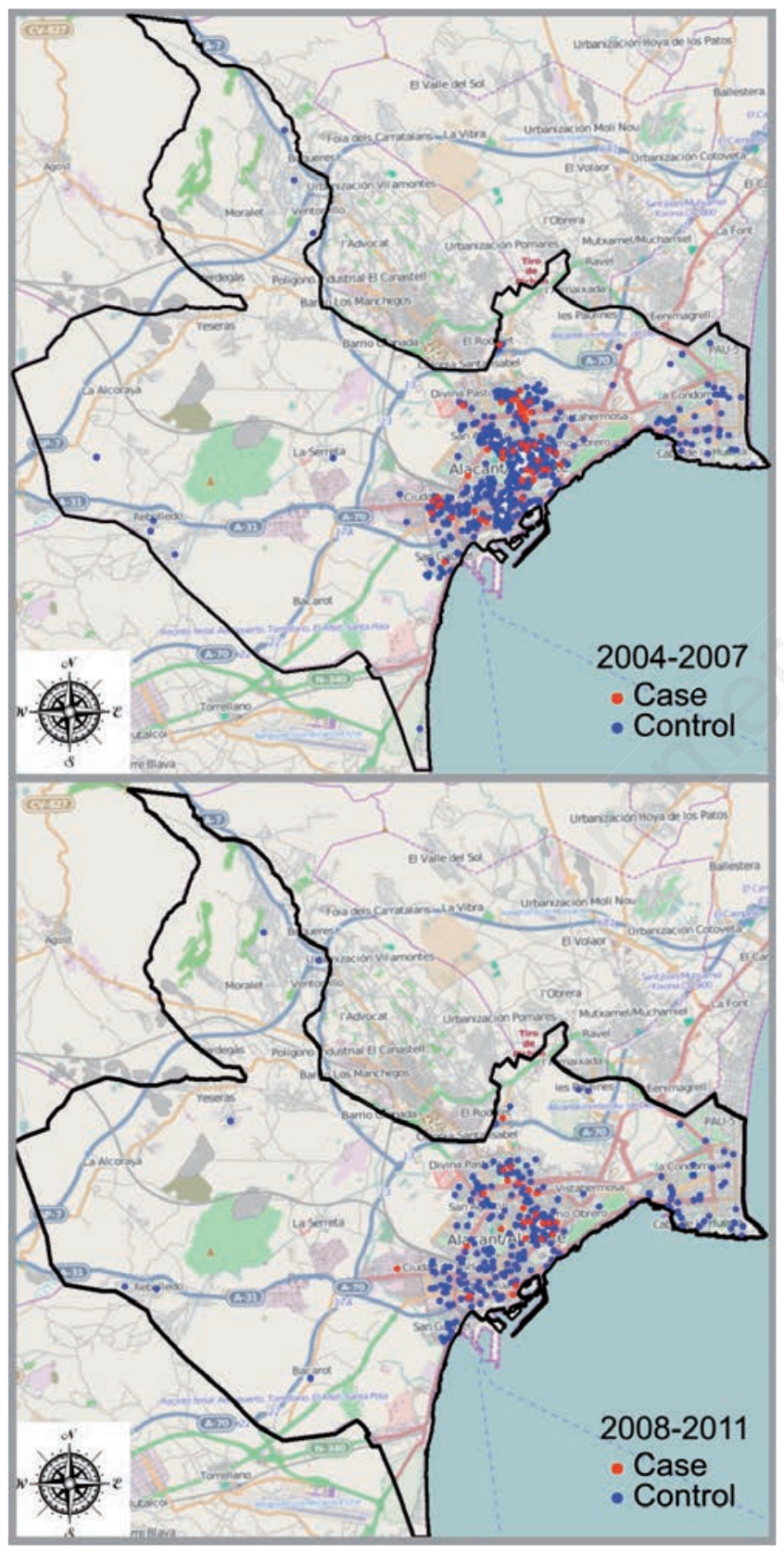

Figure 1. Locations of residents who died due to human immunodeficiency virus/acquired immunodeficiency syndrome cases (red) and controls (blue). First period (2004-2007) above and second period (2008-2011) below. Source of background map: OpenStreeMap ${ }^{\circledR}$ (http://www.openstreetmap.org/copyright/en).

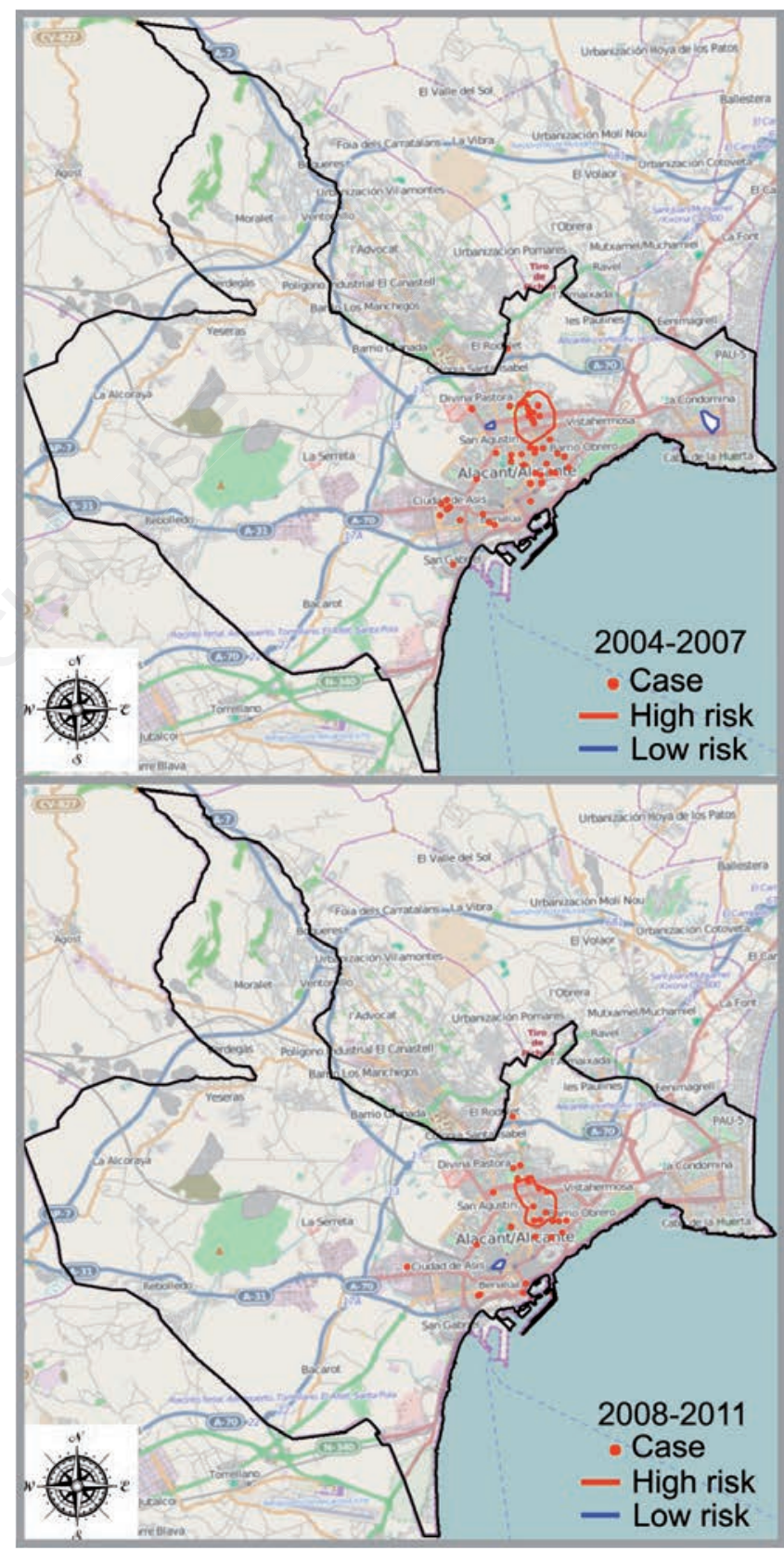

Figure 2. Areas signifying high risk (red) and low risk (blue) mortality due to human immunodeficiency virus/acquired immunodeficiency syndrome. First period (2004-2007) above and second period (2008-2011) below. Source of background map: OpenStreeMap ${ }^{\circledR}$ (http://www.openstreetmap.org/copyright/en). 
In order to estimate the intensity functions, non-parametric methods with kernel functions were used, where the NadarayaWatson (Nadaraya, 1961; Watson, 1964) estimator is:

$\hat{\lambda}(x)=\frac{\sum_{i=1}^{n} k\left(\frac{x-x_{i}}{h}\right)}{h^{2} p_{h}(x)}$

where $p_{h}(x)=\int h^{-2} k\left(\frac{x-u}{h}\right) d u$ is the edge effect correction and $k(\cdot)$ the kernel function. These functions were estimated using a point grid covering the study area giving the variation of the density (Sobj_SpatialGrid, maptools package) using the density parameter maxDim $=100$. In order to achieve the first objective of the study (to estimate a risk area in region $A$ ) the log-risk function was established by $\rho(x)=\log \left\{\frac{\lambda_{1}(x)}{\lambda_{2}(x)}\right\}$ for a grid of points $x$ on region $A$, where $\lambda_{1}$ and $\lambda_{2}$ were the intensity functions of the cases and controls, respectively, as estimated by means of the NadarayaWatson estimators described, with quartic kernel function (in this case function spkernel2d, spatstat package). The smoothing parameter $h$ was estimated through cross-validation leave one out by means of the least-square method (Kelsall and Diggle, 1998) (function $m s e 2 d$, splancs package). In order to evaluate the significance, the random labelling simulation procedure was used: under the null hypothesis, the cases and controls are generated by the same distribution. A variant of this method is applied, since the cases and controls have been matched by age and sex on a 1:10 ratio in the design. If this matching is not taken into account, the results might be biased and incorrect (Chetwynd et al., 2001). Each case is matched with 10 controls that form a tuple of 11 elements. Let $x_{i 1}, x_{i 2}, \ldots, x_{i 11}$ be the $\mathrm{i}^{\text {th }}$ tuple, then $j=1$ is the case position, and $j>1$ the position of the control group matched to the case. The procedure consists of randomly choosing an element of each tuple and label it as case and the rest of elements as controls. The case of the tuple was chosen with probability:

$$
p_{i}=p\left(x_{i 1}\right) / \sum_{j=1}^{11} p\left(x_{i j}\right)
$$

where $p(x)$ is the probability that an individual in the location $\mathrm{x}$ is a case (Diggle, 2003). Once each case/control was established, $\rho_{j}(x)$ was calculated in each step and repeated for $j=1, \ldots, 100$ simulations. In this way, the contours of higher risk were obtained, i.e. the areas where the proportions of values observed being higher than the simulated ones, were higher than the 0.975 percentile. The areas with a lower risk of cases were those where the proportions of values observed being lower than the simulated ones, were higher than the 0.975 percentile.

In order to achieve the second objective of the study (to analyse the spatial evolution of the risks of death over time) the approach proposed by Diggle et al. (2005) was adopted. The areas at risk $\rho_{j}(x)$ were estimated for each discrete period of time $t$ by choosing the same smoothing parameter in both periods of time, which was estimated through the least square method for all the events of both periods together. In this case, the null hypothesis was that there are no changes over time in the areas at risk, i.e. Ho: $\rho_{t}(x)=\rho(x)$ for $t=1,2$. The statistic is:

$$
T=\sum_{t} \sum_{x \in t}\left[\hat{\rho}_{t}(x)-\bar{\rho}(x)\right]^{2}
$$

where $\bar{\rho}(x)$ is the average risk of both periods of time in each location $x$ of $A$. The $\mathrm{P}$ value is obtained by random labelling, where each period is randomly assigned to each case in each simulation, and the number of original cases is constant. Also, in this case, each control matched by group of age and sex was assigned to each case. A total of 1000 simulations were carried out, all the $T_{j}$ values were calculated for $j=1, \ldots 1000$, and the $P$ value estimated by the Monte Carlo method, which determines that, in the case of $\mathrm{Ho}$ being true, the probability of rejecting $H o$ is given by the proportion of $\mathrm{Tj}>\mathrm{To}+1$, with $\mathrm{j}=1, \ldots \mathrm{m}+1$ being $T o$ as statistically estimated from the observed data. In order to obtain random samples of the control groups, the statistical programme SPSS v18 was used (SPSS-Inc., 2009). All the analyses and mappings were made through the statistical environment R v3.0.3 (R Core Team, 2014). The background map of the municipal area of Alicante was downloaded through the $\mathrm{R}$ package OpenStreetMap, and is available under the Open Database License (Open Knowledge Foundation, 2015b). Any rights in individual contents of the database are licensed under the Database Contents License (Open Knowledge Foundation, 2015a).

\section{Results}

A total of 50 and 27 deaths due to HIV/AIDS in the study area were identified for the first and second period, respectively. The control groups were made up by 500 and 270 controls, respective-

Table 1. Frequency of death by human immunodeficiency virus/acquired immunodeficiency syndrome in the municipal area of Alicante, Spain, by age group and period.

\begin{tabular}{lcc}
\hline Age group (years) & $2004-2007$ & $2008-2011$ \\
$25-29$ & 1 & 1 \\
$30-34$ & 10 & 2 \\
\hline $35-39$ & 9 & 2 \\
$40-44$ & 12 & 4 \\
\hline $45-49$ & 9 & 4 \\
$50-54$ & 0 & 7 \\
\hline $55-59$ & 2 & 1 \\
$60-64$ & 4 & 3 \\
\hline $65-69$ & 0 & 1 \\
$70-74$ & 1 & 1 \\
\hline $75-79$ & 2 & 0 \\
$80-84$ & 0 & 1 \\
\hline Total & 50 & 27 \\
Descriptive statistics for age & & \\
Minimum (years) & 26 & 29 \\
Maximum (years) & 77 & 49.4 \\
Mean (years) & 43.7 & 11.9 \\
Standard deviation & 11.7 & \\
\hline
\end{tabular}


ly. Table 1 shows the frequency of death by age group and descriptive statistics for age. Figure 1 shows the spatial distribution of the cases (red) and controls (blue) for the periods 2004-2007 and 2008-2011, respectively. Figure 2 shows the location of the cases and estimated risk areas for each period, including $95 \%$ confidence bands. The red bands show the areas with the highest clustering of cases compared to the controls, and thus the areas with a significant increased risk of death due to HIV/AIDS. The blue bands show the areas with a lower significant risk of death. High-risk areas were located during both periods around neighbourhoods named Virgen del Remedio, Virgen del Carmen, Cuatrocientas Viviendas, Colonia Requena and Juan XXIII. There were small areas with a lower risk in Cabo de las Huertas for the first period, and near the Centro for the second. The test for area variation with respect to risk between the two periods provided a $\mathrm{P}$ value of 0.9660 . Therefore, there is no evidence of a significant spatial change of the areas at risk in both periods studied.

\section{Discussion}

This study proves that the methodology of point processes is a useful and effective tool to analyse mortality space-time patterns in cities, and thus can complement aggregate data methods when the number of cases is low. High-risk areas that can be perfectly identified and located in the city were detected in both periods. This area has one of the lowest socioeconomic levels in the city and remains the same from the first period of time studied to the second. To our knowledge, this is the first study that applies point process methodology to analyse mortality due to AIDS/HIV in urban areas. As expected, areas with a high risk of death from AIDS/HIV were located in the most deprived areas of the city. A previous study had analysed mortality due to different preventable causes in this city for the period 1996-2003 using aggregate data by census sections (Nolasco et al., 2009), although a different methodology was used. The results obtained from this study are similar to the ones obtained in the aforementioned study on HIV/AIDS, since a higher risk of death was found also in the census sections with a lower socioeconomic level compared to those with a higher socioeconomic level. Furthermore, the census sections that were detected in the former study approximately coincide with the area of significant excess risk detected in this study. The results described in the literature also coincide: mortality due to HIV/AIDS is associated with a lower socioeconomic level (Salgado-Barreira et al., 2014). From period to period, mortality due to AIDS/HIV in men in the city of Alicante decreased by $46 \%$. By contrast, in the Valencian Community and Spain it decreased by 22 and $25 \%$, respectively, during the same periods (I.N.E., 2014). These results and the significant increase in the age of death in the second period compared to the first in almost 6 years was to be expected, because the Spanish National Health System successfully introduced the treatment with highly active antiretroviral therapy in 1996, which became free and accessible to everyone. The results of this study prove that the area of significant risk of death due to AIDS/HIV remains the same from one period to the other around the run-down area. In a study carried out in the city of Barcelona, Borrell and colleagues also found that inequalities due to socioeconomic level remained the same over time in HIV/AIDS mortality (Borrell et al., 2006).
This study considers two periods of time. The first period takes place before the global economic crisis, which had a high impact in Spain, until 2007. The second period takes place during the economic crisis, as of 2008. Thus, it appears that the economic crisis did not affect the spatial patterns of AIDS/HIV mortality in the city of Alicante; nonetheless, the last year comprised by this study was 2011, the effects of the crisis might be reflected in subsequent years. Some studies proved that certain trends in the inequalities in mortality might be related to the lack of access to the highly active antiretroviral therapy by the population groups with a lower socioeconomic level. This fact suggests that attention should be paid to the application of economic control measures so that the access to this therapy is not affected.

In addition to the access to specific therapies, the mortality analysed in this study is avoidable, or at least reducible, through health programmes and policies in the field of primary prevention. The identification of areas carrying higher risk should facilitate concrete actions in terms of health promotion and protection, as well as health education.

The main limitation of the study is that the individual characteristics of the subjects studied were not been included. The methodology used allows including various explanatory variables. A future research direction should include confounding features and/or individual risk factors, such as socioeconomic level, lifestyle, diet, genetic aspects, etc., and include them in models that take into account covariates, such as generalised additive models (Kelsall and Diggle, 1998), or through intensity functions (Diggle et al., 2007). The design of case-control matched by five-year age groups and separated by sex used in this study guarantees the control of these two clearly confounding variables in mortality patterns. Future studies may also analyse the continuous interaction of space-time for inhomogeneous point processes (Gabriel and Diggle, 2009; Moller and Diaz-Avalos, 2010).

As regards the strengths of the study, it is worth mentioning that a list of the population containing all the individuals that make up the population at risk was available, covering approximately 330,000 inhabitants per year, which was useful for obtaining random samples stratified and matched by age and sex to create the control groups. In contrast to other studies, we used here representative controls from people alive, which perfectly represent the spatio-temporal structures of the population at risk. Similarly, information about all deaths occurring in the area of study was available thanks to the Register of Mortality of the Valencian Community that was available as a source of information. This register includes precisely all occurring deceases.

\section{Conclusions}

Point processes are useful and effective to study mortality in urban areas, and a complement to aggregate data methods. Mortality due to HIV/AIDS shows a higher concentration of risk in financially deprived areas of the city. The clustering pattern remained the same in the two periods of time studied: 2004-2007 and 2008-2011, which indicates that stronger interventions are needed. Further analysis should be done taking into account socioeconomic or environmental variables, as well as other risk factors. 


\section{References}

Besag J, York J, Mollie A, 1991. A Bayesian image restoration, with two applications in spatial statistics. Ann I Stat Math 43:1-21.

Birc JM, Alexander FE, Blair V, Eden OB, Taylor GM, McNally RJ, 2000. Space-time clustering patterns in childhood leukaemia support a role for infection. Brit J Cancer 82:1571-1576.

Borrell C, Rodriguez-Sanz M, Pasarin MI, Brugal MT, Garcia-deOlalla P, Mari-Dell'Olmo M, Cayla J, 2006. AIDS mortality before and after the introduction of highly active antiretroviral therapy: does it vary with socioeconomic group in a country with a National Health System? Eur J Public Health 16:601-8.

Chetwynd AG, Diggle P, Marshall A, 2001. Investigation of spatial clustering from individually matched case-control studies. Biostatistics 2:277-93.

Diggle P, 2003. Statistical analysis of spatial point patterns. 2nd ed. Arnold, London, UK.

Diggle P, Chetwynd AG, 1991. Second-order analysis of spatial clustering for inhomogeneous populations. Biometrics 47:1155-63.

Diggle P, Gomez-Rubio V, Brown PE, Chetwynd AG, Gooding S, 2007. Second-order analysis of inhomogeneous spatial point processes using case-control data. Biometrics 63:550-7.

Diggle P, Rowlingson B, 1994. A conditional approach to point process modelling of elevated risk. J Roy Stat Soc A 157:433-40.

Diggle P, Rowlingson B, Su TI, 2005. Point process methodology for on-line spatio-temporal disease surveillance. Environmetrics 16:423-34.

Diggle P, Zheng P, Durr P, 2005. Nonparametric estimation of spatial segregation in a multivariate point process: bovine tuberculosis in Cornwall, UK. J Roy Stat Soc C 54:645-58.

Diggle PJ, 2006. Spatio-temporal point processes, partial likelihood, foot and mouth disease. Stat Methods Med Res 15:325-36.

Gabriel E, Diggle P, 2009. Second-order analysis of inhomogeneous spatio-temporal point process data. Stat Neerl 63:43-51.

Gelman A, Price PN, 1999. All maps of parameter estimates are misleading. Stat Med 18:3221-34.

Google, 2014. Geocoding API. Available from: https://developers.google.com/maps/documentation/geocoding/start?hl=en

INE, 2014. Instituto Nacional de Estadística. Available from: www.ine.es

Kelsall JE, Diggle P, 1995. Non-parametric estimation of spatial variation in relative risk. Stat Med 14:2335-42.

Kelsall JE, Diggle P, 1998. Spatial variation in risk of disease: a nonparametric binary regression approach. J Roy Stat Soc C 47:559-73.

Kulldorff M, 1997. A spatial scan statistic. Commun Stat 26:148196.

Lawson AB, Clark A, 2002. Spatial mixture relative risk models applied to disease mapping. Stat Med 21:359-70.

Linares S, Tisnés A, Nochera A, 2008. Aplicación de Sistemas de Información Geográfica para detectar patrones espaciales en la mortalidad por cáncer en la ciudad de Tandil. In: M.C. García et al. (eds.) Geografía Argentina. Aportes al estudio de algunos problemas actuales. Centro de Investigaciones Geográficas, Tandil, Argentina, pp. 63-9.

López-Abente G, Ibáñez C, 2001. Aplicación de técnicas de análisis espacial a la mortalidad por cancer en Madrid. Comunidad autónoma, Consejería de Sanidad, Dirección General de Salud Pública, Madrid, Spain.
Mackenbach JP1, Hoffmann R, Khoshaba B, Plug I, Rey G, Westerling R, Pärna K, Jougla E, Alfonso J, Looman C, McKee M, 2013. Using 'amenable mortality' as indicator of healthcare effectiveness in international comparisons: results of a validation study. J Epidemiol Commun Health 67:139-46.

Moller J, Diaz-Avalos C, 2010. Structured spatio-temporal shotnoise Cox point process models, with a view to modelling forest fires. Scandin J Stat 37:2-25.

Nadaraya EA, 1961. On estimating regression. Theory Prob Appl 9:141-2

Nolasco A1, Melchor I, Pina JA, Pereyra-Zamora P, Moncho J, Tamayo N, García-Senchermes C, Zurriaga O, MartínezBeneito MA, 2009. Preventable avoidable mortality: evolution of socioeconomic inequalities in urban areas in Spain, 19962003. Health Place 15:702-11.

Nolte E, McKee M, 2004. Does health care save lives? Avoidable mortality revisited. Nuffield Trust, London, UK.

Open Knowledge Foundation, 2015a. Database Contents License (DbCL) v1.0. Available from: http://opendatacommons. org/licenses/dbcl/1.0/

Open Knowledge Foundation, 2015b. Open Database License (ODbL) v1.0. Available from: http://opendatacommons. org/licenses/odbl/1.0/

Quesada JA, Nolasco A, Moncho J, 2013. Comparison of Google and Yahoo applications for geocoding of postal addresses in epidemiological studies. Rev Esp Salud Publica 87:201-6.

R Core Team, 2014. R: A language and environment for statistical computing. R Core Team, Vienna, Austria. Available from: http://www.r-project.org/

Raskin P, Kemp-Benedict E, 2004. Global environment outlook scenario framework: background paper for UNEP's third global environment outlook report (GEO-3). Division of Early Warning and Assessment, United Nations Environment Programme, Nairobi, Kenya.

Regidor E, Sanchez E, de la Fuente L, Santos JM, Martinez D, 2011. A proposal of measures for monitoring social disparities in health using AIDS and liver disease mortality before and after HAART. Eur J Public Health 21:116-21.

Richardson LA, Milloy MJ, Kerr TH, Parashar S, Montaner JS, Wood E, 2014. Employment predicts decreased mortality among HIV-seropositive illicit drug users in a setting of universal HIV care. J Epidemiol Commun Health 68:93-6.

Richardson S, Thomson A, Best N, Elliott P, 2004. Interpreting posterior relative risk estimates in disease-mapping studies. Environ Health Persp 112:1016-25.

Salgado-Barreira A, Estany-Gestal A, Figueiras A, 2014. Effect of socioeconomic status on mortality in urban areas: a systematic critical review. Cad Saude Publica 30:1609-21.

Shao C, 2011. Approaches to the spatial modelling of cáncer incidence and mortalityu in metropolitan Perth, Western Australia, 1990-2005. Edith Cowan University, Joondalup, Australia. Available from: http://ro.ecu.edu.au/cgi/viewcontent.cgi?article $=1422 \&$ context $=$ theses

SPSS-Inc., 2009. PASW Statistics for Windows, Version 18.0. SPSS Inc., Chicago, IL, USA. Retrieved from: http://www01.ibm.com/software/analytics/spss/

Watson GS, 1964. Smooth regression analysis. Indian J Stat A 26:359-72.

Wheeler DC, 2007. A comparison of spatial clustering and cluster detection techniques for childhood leukemia incidence in Ohio, 1996-2003. Int J Health Geogr 6:13. 